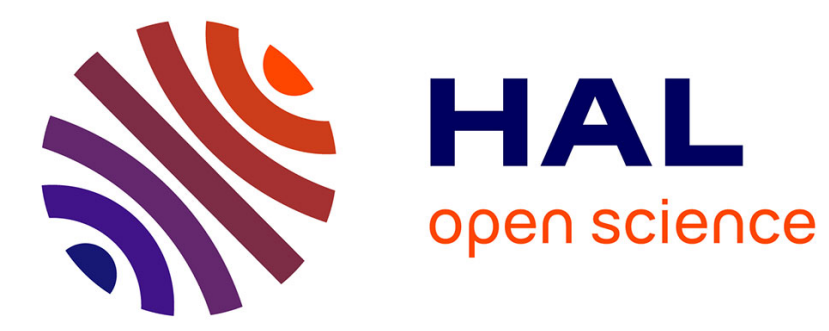

\title{
Noncanonical Functions of Antibodies
}

\author{
Jordan D Dimitrov, Sébastien Lacroix-Desmazes
}

\section{To cite this version:}

Jordan D Dimitrov, Sébastien Lacroix-Desmazes. Noncanonical Functions of Antibodies. Current Trends in Immunology, 2020, 41 (5), pp.379-393. 10.1016/j.it.2020.03.006 hal-03031776

\section{HAL Id: hal-03031776 https://hal.science/hal-03031776}

Submitted on 18 Dec 2020

HAL is a multi-disciplinary open access archive for the deposit and dissemination of scientific research documents, whether they are published or not. The documents may come from teaching and research institutions in France or abroad, or from public or private research centers.
L'archive ouverte pluridisciplinaire HAL, est destinée au dépôt et à la diffusion de documents scientifiques de niveau recherche, publiés ou non, émanant des établissements d'enseignement et de recherche français ou étrangers, des laboratoires publics ou privés. 


\section{non-canonical functions of antibodies}

Jordan D. Dimitrov and Sébastien Lacroix-Desmazes

Centre de Recherche des Cordeliers, INSERM, Sorbonne Université, USPC, Université Paris Descartes, Université Paris Diderot, F-75006 Paris

\section{Address for correspondence:}

INSERM UMRS 1138,

Centre de Recherche des Cordeliers,

75006 Paris, France.

Tel: +33144278206

Fax: +33144278194

E-mail: jordan.dimitrov@crc.jussieu.fr / jordan.dimitrov@inserm.fr 


\begin{abstract}
The canonical functions of antibodies are based on joining the process of antigen recognition with initiation of innate immune reactions. With the introduction of modern research approaches, and the use of sophisticated model systems, the recent years have witnessed the discovery of numbers of non-canonical functions of antibodies. These functions encompass either untypical strategies for neutralization of pathogens or exertion of activities by antibodies that are typically characteristic for other proteins (cytokines, chaperons or enzymes). Here we provide an overview of non-canonical functions of antibodies and discuss their mechanisms and implications for immune regulation and immune defence. A better comprehension of these functions will enrich our knowledge about the adaptive immune response and shall inspire the development of novel therapeutics.
\end{abstract}




\section{Highlights}

Antibodies are major player in adaptive immunity. They participate in immune defence and have potent immune-modulatory potential.

Antibody molecules have two functional centres - they bridge the specific antigen recognition through antigen-binding site with interaction and activation of innate immune receptors, via their constant regions. These integrated interactions are essential for the classical antibody functions.

Antibodies can utilize alternative or non-canonical strategies for broadening of their functional competency. Thus antibodies perform untypical activities by acquiring activities typical for cytokines, chaperones, transporters, enzymes etc.

The non-canonical functions of antibodies contribute to the immune defence and the maintenance of physiological homeostasis. Many of these activities are operational in healthy individuals. Others appear only as result of immunopathology.

The non-canonical functions of antibodies arise as a result of enormous diversity of adaptive immune repertoires and micro-evolutionary selection of B cells. They provide a rich source of potential therapeutic modalities. 


\section{The structure determines the functions of antibodies}

Antibodies are protagonists of the adaptive immunity with key roles in immune defence and in immune regulation. They are produced by B lymphocytes, which undergo complex lifecycle that culminates in differentiation into antibody-secreting plasma cells. The characteristic molecular organization of immunoglobulin molecules, namely the presence of two functionally distinct poles: an antigen-binding site and a constant region (BOX 1), determines most of the biological functions of antibodies. Thus, the prototypical property of antibodies is their potential to link antigen recognition, mediated by the variable regions, with engagement of different types of innate immune proteins ( $\mathrm{Fc}$ receptors $(\mathrm{FcR}), \mathrm{C} 1 \mathrm{q}$, TRIM21), through the constant region. These interactions trigger diverse sets of biological reactions (Figure 1) (Lu et al., 2018).

Although relatively invariant as compared to the variable region, the constant (or Fc) regions of different antibody molecules display certain heterogeneity. Thus, as based on sequence differences in the constant portion of the heavy chain, five immunoglobulin classes are identified in humans - $\operatorname{IgG}, \operatorname{IgA}, \operatorname{IgM}, \operatorname{IgD}$ and $\operatorname{IgE}$ (Schroeder and Cavacini, 2010, Vidarsson et al., 2014). Moreover, IgG and IgA classes are subdivided into subclasses - IgG1, IgG2, IgG3 and IgG4 as well as IgA1 and $\operatorname{IgA} 2$, respectively. Each immunoglobulin class or subclass has preferential binding to different types of receptors for the constant region ( $\mathrm{FcR})$. These receptors are differentially expressed on different cell types, and hence their engagement leads to distinct biological reactions (Bournazos et al., 2017). The immunoglobulin classes and subclasses also vary in their potential to interact with plasma protein $\mathrm{C} 1 \mathrm{q}$ and consequently activate the complement system. The diversity of Fc portions is further 
extended by variations in the glycosylation patterns and existence of Fc sequence polymorphisms (BOX 2). This heterogeneity leads to further fine-tuning of the functional activity of antibodies.

Typical biological functions (Figure 1) can be attributed to particular antibody classes of heavy chain. For example, characteristic functions of $\operatorname{IgG}$ upon recognition of the pathogen and engagement of Fc $\gamma \mathrm{R}$ include antibody-dependent cellular cytotoxicity (ADCC), antibody-dependent cellular phagocytosis (ADCP) or induction of production of reactive oxygen species and pro-inflammatory cytokines. Antibodies from the $\operatorname{IgG}$ class have also the capacity to activate complement or direct antigens towards degradation by proteasome, through interactions with C1q and TRIM21, respectively (Diebolder et al., 2014, McEwan et al., 2013). On the other hand, the antibodies belonging to $\operatorname{IgM}$ class are the most proficient activators of the complement system (Sharp et al., 2019). The antigen recognition by $\operatorname{IgE}$ bound to FceRI initiates cellular degranulation and release of biologically active substances. Alternatively, serum IgA performs its biological activities of immune regulation and immune defence mainly through interactions with FcaRI expressed on myeloid cells (Mkaddem et al., 2014, van Egmond et al., 2000).

The diversity of biological outcomes elicited by the canonical antigen-antibody-innate receptor triad allows tailoring the reaction to the idiosyncratic characteristics of each pathogen. Recent studies suggested that even antibody activities that were considered independent of constant region, such as neutralization of toxins and viruses, rather involve the innate receptor systems (Abboud et al., 2010, Bournazos et al., 2014a, Bournazos et al., 2014b). Nevertheless, there is accumulating evidence for the existence of alternative functions of antibodies that do not fit in the classical triad antigen-antibody-innate receptor. Thus, by using only variable region antibodies can 
perform functions typical for other proteins (enzymes, cytokines, chaperones) or employ non-conventional mechanisms inactivation of pathogens or immune regulation. The aim of the present review is to present the current state-of-the-art about the non-canonical functions of antibodies and to highlight their importance for our basic understanding of the immune system, and as potential therapeutic tools.

\section{Non-canonical functions of antibodies}

Non-canonical functions of antibodies can be regarded as those where the classical triad antigen-antibody-innate receptor is disconnected (Figures 2 and 3). These functions are predominantly exerted by the variable region. They emerge as a consequence of the great diversity of sequences and topological configurations of the antigen-binding site, which is shaped by both genetic and post-translational mechanisms (Kanyavuz et al., 2019). The non-canonical functions of antibodies can contribute to immune defence and to immune regulation. Some of the antibody functions are implicated in both processes.

\section{Non-canonical functions of antibodies important for immune defence}

\section{Antibody-mediated catalysis}

The existence of antibodies with enzymatic activity was foreseen during pioneering studies of immunoglobulin structure. Catalysis is a typical example of a nonconventional antibody activity that emerges as a result of the stochastic nature of generation the sequence diversity of antigen-binding site. Thus, antigen-binding sites of some antibodies display a constellation of residues that is similar to that found in the active sites of enzymes and, hence, can catalyse the corresponding chemical transformations. Large numbers of investigations in the last 30 years have confirmed 
that antibodies with different catalytic activities are generated by immunization with appropriate reaction intermediates (Xu et al., 2004). These studies, however, also revealed that catalytic efficiency achieved by antibodies is considerably inferior to that of their natural enzyme counterparts.

Besides rationally elicited catalytic antibodies, antibodies with natural catalytic activity are also found in immune repertoires under physiological and pathological conditions (Bowen et al., 2017a). In the human, the most frequently detected catalytic antibodies exhibit proteolytic activity. These antibodies can bind and hydrolase pathogen-specific target molecules, thus potentially contributing to the immune defence. Indeed, proteolytic antibodies belonging to different classes (IgM, IgA and IgG) and isolated from healthy humans were demonstrated to hydrolyse envelope proteins of HIV-1 (Paul et al., 2004, Planque et al., 2007). Antibodies that catalyse the degradation of molecules of bacterial or fungal pathogens were also characterized (Bowen et al., 2017b, Hifumi et al., 2008). A recent study has described an antibody with dual hydrolytic activity - proteolytic and glycolytic. This antibody specifically binds to the surface of fungal pathogen Cryptococcus neoformans and induces the release of capsular polysaccharides, thus directly contributing to pathogen inactivation (Bowen et al., 2017b). Notably, in the same study, structural bioinformatics demonstrated that approximately $7 \%$ of 1602 antibodies with available crystal structure possess a serine protease catalytic triad that can eventually exert proteolytic activity. This result suggests that the low catalytic efficacy of proteolytic antibodies can be compensated by their high frequency, thus conferring them with physiological relevance. The significance of catalytic antibodies in immune defence against bacteria was also inferred by a study showing a positive correlation of the 
titers of proteolytic antibodies with the survival of patients with severe sepsis (Lacroix-Desmazes et al., 2005).

Antibodies with proteolytic activity have been also reported in neurodegenerative, autoimmune and alloimune diseases (Lacroix-Desmazes et al., 2002, Li et al., 1995, Paul et al., 1989, Ponomarenko et al., 2006, Pradhan et al., 2018, Wootla et al., 2011, Wootla et al., 2008, Yang et al., 2010). It was suggested that proteolytic antibodies have both detrimental and beneficial effects under these conditions. Their poor catalytic efficiency and the concurrent presence of antibodies using other mechanisms makes difficult to evaluate the real contribution of catalytic antibodies in different disease conditions or in immune defence. Nonetheless some studies has investigated the therapeutic potential of proteolytic antibodies (Planque et al., 2015).

\section{Direct pathogen inactivation}

Antibodies usually kill microbes by induction of complement-mediated lysis or phagocytosis by FcR-bearing innate immune cells (Lu et al., 2018). However, numerous studies identified monoclonal antibodies (mostly belonging to $\operatorname{IgM}$ and $\operatorname{IgG}$ isotypes) that can induce microbe killing in the absence of effector systems (Casadevall and Pirofski, 2011, Coleman et al., 1992, Connolly et al., 2004, LaRocca et al., 2008, Mukherjee et al., 1995, Tian et al., 2009). Antibodies specific for diverse species of pathogenic bacteria and fungi thus induce direct killing (Figure 2). However, the mechanistic aspects of direct antibody-mediated microbicidal activity were elucidated only in few cases. In case of the fungal pathogen Cryptococcus neoformans, it was demonstrated that two directly inactivating monoclonal $\operatorname{IgM}$ and IgG induce changes in pathogen gene expression and metabolism upon binding to the cell surface (McClelland et al., 2010). The study revealed that the two monoclonal 
antibodies bind to different sites on the capsule and trigger distinct changes in gene expression and metabolism. The induced alteration in fungal metabolism may explain the ability of these antibodies to inhibit directly polysaccharide release and biofilm formation. Another study investigated the mechanism of direct inhibitory effect of antibodies specific for pneumococcal capsular polysaccharide of Streptococcus pneumoniae (Yano et al., 2011). These antibodies were highly protective in vivo despite their inability to trigger Fc-dependent killing in vitro. The binding of the directly killing antibodies to the bacterial surface also induced changes in gene expression that caused interference with bacterial quorum sensing finally resulting in augmented bacterial agglutination and fratricide activity. Other investigations depicted the mechanism of direct antibody mediated killing of Borrelia burgdorferi, the pathogen causing Lyme disease. Some antibodies induce killing of the pathogen in absence of complement. It is noteworthy that Fab fragment these antibodies have similar bactericidal activity as intact antibody (Coleman et al., 1992, Sadziene et al., 1994). The monoclonal IgG1 antibody CB2 specifically recognizes protein OspB on the bacterial surface. The binding of this antibody causes release of membrane vesicles and formation of large pores $(2.8-4.4 \mathrm{~nm})$ in the pathogen outer membrane, resulting in killing of bacteria by osmotic shock (LaRocca et al., 2009). The direct bactericidal activity of CB2 was further demonstrated to depend on the presence of cholesterol and cholesterol glycolipids in the membrane of Borrelia burgdorferi (LaRocca et al., 2010). The cholesterol and cholesterol glycolipids were found to cluster around the target antigen of $\mathrm{CB} 2$, OspB, thus forming lipid rafts. Hence, the cholesterol may facilitate antibody-induced release of membrane vesicles that consequently contribute to formation of pores and killing of bacteria. In a recent study, yet another mechanism of direct bactericidal effect by antibody was described (Storek 
et al., 2018). BamA is $\beta$-barrel assembly machine on the outer membrane of gramnegative bacteria responsible for folding and assembly of outer membrane proteins. A monoclonal IgG antibody specific for BamA from Escherichia coli blocks the folding activity of the protein, thus resulting in accumulation of unfolded proteins in the periplasmic space and disruption of the bacterial membrane integrity. These effects induced by antibody binding and blocking of the activity of key protein folding machinery on the outer membrane finally result in bacterial killing (Storek et al., 2018).

The accumulated mechanistic evidence highlights the diversity of the mechanisms exploited by antibodies to directly inactivate pathogens. A common feature of these antibodies is the recognition of vulnerable targets on the microbe surface leading to alteration of essential biological processes. The existence of such antibodies has important consequences from an evolution point of view. In addition to supplementing the canonical bactericidal activities of antibodies, such properties may counteract the ability of some bacteria to block the formation of the membrane attack complex by the complement system, or to evade Fc-receptor recognition through $\mathrm{Fc}$ fragment removal by bacterial proteases. Indeed, the binding of Fab fragments would be sufficient for induction of direct bacterial inactivation.

\section{Non-canonical functions of secretory $\operatorname{IgA}$}

Secretory IgA (sIgA) plays a significance immune protective role at epithelial surfaces as strongly implied by the fact that $\operatorname{sig} \mathrm{A}$ is the most highly produced immunoglobulin in humans (>4 g/day). The well-known mechanisms of immune defence by sIgA involve bacterial opsonisation, blocking of virulence factors, induction of bacterial agglutination as well as direct neutralization of viruses and 
toxins (Macpherson et al., 2018). The recent years have witnessed a large surge of interest towards mucosal immunity and the role of the intestinal microbiota in human physiopathology. Some studies have uncovered ingenious functions of $\operatorname{sIgA}$ in immune defence and regulation that go well beyond their classically ascribed roles

\section{(Figure 2).}

Some of the recently described non-conventional functions of sIgA emerged as a consequence of the pronounced antigen-binding promiscuity of mucosal $\operatorname{IgA}$. The capacity of a single antibody clone to recognize multiple structurally unrelated antigens with physiologically relevant binding affinity is referred to as polyreactivity (Dimitrov et al., 2013). Polyreactive antibodies represent normal constituent of immune repertoires in healthy humans. Indeed, ca. $20 \%$ of human memory peripheral B cells express polyreactive antibodies (Berkowska et al., 2015, Tiller et al., 2007). Notably, the frequency of B cells in humans and mouse expressing polyreactive antibodies is significantly greater in the intestinal mucosa (Benckert et al., 2011, Bunker et al., 2017). Thus, approximately $66 \%$ of $\operatorname{IgA}+\mathrm{B}$ cells isolated from the small intestine and $57 \%$ of $\operatorname{IgA}+\mathrm{B}$ cells isolated from the colon of mice display polyreactivity. In contrast, $28 \%$ of conventional splenic B2 cells exhibit polyreactivity (Bunker et al., 2017). Importantly, a significant fraction of sIgA originates from thymus-independent immune responses (predominately derived from B1b B cells in mice). These sIgA have the potential to coat most of bacterial species that are normally recognized by mucosal antibodies in the small intestine (Bunker et al., 2015), suggesting that intestinal polyreactive has a very broad epitope coverage, that overlaps with bacterial recognition by the repertoire of thymus-dependent sIgA.

One of the recently described non-canonical functions of $\operatorname{sg} \mathrm{A}$ that depend on polyreactivity, is the ability to mediate trans-epithelial transfer of bacteria in the small 
intestine (Fransen et al., 2015). The actual mechanism of transfer of the IgA-coated bacteria is not understood, and is independent from Dectin-1-mediated transfer of sIgA immune complexes through M-cells (Rochereau et al., 2013) or through phagocytosis by $\mathrm{CX} 3 \mathrm{CR} 1+$ mononuclear phagocytes. Yet, the transfer of bacteria through the intestinal epithelia has important consequences (Fransen et al., 2015). It initiates T-cell-dependent antibody responses in the gut-associated lymphoid tissues (Peyer's patches) and generates bacteria-specific antibodies. It is noteworthy that the coating of bacteria by polyreactive antibodies allows generation of specific mucosal immune responses toward non-invasive bacterial species that would normally not be able to breach the epithelial barrier and prime the immune system. In this context, polyreactive $\operatorname{sg} \mathrm{A}$ thus function as an adjuvant to prime humoral immune responses (Fransen et al., 2015). The same study reported a marked difference in the basic level of polyreactive intestinal sIgA among strains of mice. Naïve BALB/c mice have considerably higher levels of polyreactive sIgA as compared to C57BL/6 mice that translates into different potentials for trans-epithelial bacterial transfer (Fransen et al., 2015). Interestingly, the levels of polyreactive sIgA positively correlated with the diversity of the microbiota in the two strains of mice. Thus, it was proposed that the trans-epithelial bacterial transfer mediated by innate polyreactive sIgA primes bacteria-specific immune responses that in turn play a central role in controlling bacterial diversity in the microbiota. This observation is in line with another study highlighting the importance of bacterial recognition by specific sIgA for the successful colonization of the intestine by bacteria (Donaldson et al., 2018).

Another recently identified non-conventional function of $\operatorname{sIg} \mathrm{A}$ is the regulation of the penetration of bacterial metabolites in the host (Uchimura et al., 2018) (Figure 2). Experiments with germ-free animals revealed that the introduction of isotope-labelled 
bacteria $(E$. coli) results in the transfer of hundreds of bacterial metabolites to all organs and body fluids. The absence of sIgA resulted in significantly higher levels of penetration of metabolites in the host tissues. $\operatorname{sg} \mathrm{A}$ were further demonstrated to control the metabolite exposure of the host by reducing the motility of bacteria through interaction with flagella (Uchimura et al., 2018). Thus, $\operatorname{sIgA}$ increase the bacterial intestinal transition and thereby reduce the time of residence in the small intestine where penetration of most of bacteria-derived metabolites takes place. Moreover, sIgA could directly interact with some of the bacterial metabolites, potentially resulting in metabolite retention in the intestinal lumen (Uchimura et al., 2018). The same study demonstrated that bacterial metabolites have a strong impact on the host by modulating inflammation and metabolism. Hence, their penetration would have systemic effects on the host immune system. Therefore, sIgA, by affecting the rate of bacterial transport through the gastrointestinal tract, may modulate, at the systemic level, the host metabolism and immunity - an example of function of antibodies that extends far beyond mere binding and neutralization.

In addition to trans-epithelial transfer of bacteria and regulation of metabolite penetration, another function of $\operatorname{sg} \mathrm{A}$ has been described. This function, however, depends on high-avidity bacterial recognition by specific antibodies (Moor et al., 2017). Vaccination of mice with Salmonella enterica serovar Typhimurium induces high avidity pathogen-specific sIgA that contribute to immune defence by formation of bacterial clumps (Moor et al., 2017) following the challenge of pre-vaccinated animals with the pathogens. In contrast to typical antibody-mediated agglutination, which also results in formation of clumps of bacteria (Hendrickx et al., 2015), this newly described mechanism is operational at low bacterial densities $\left(<10^{7} \mathrm{CFU} \cdot \mathrm{ml}^{-1}\right)$. Indeed, clumping of bacteria by high-avidity $\operatorname{sgA}$ occurs at the time of division and 
daughter cells are conjugated by antibody before separation, leading to a process termed "enchained growth" (Figure 2). It was proposed that the process of "enchained growth" accelerates transition of bacteria through the small intestine (Moor et al., 2017), thus preventing intestinal inflammation and possibly reducing penetration of immunomodulatory metabolites as described above (Uchimura et al., 2018). In addition, the restriction of bacteria in clumps diminished the clonal diversity of the bacteria by reducing the horizontal exchange of genetic material between bacteria and by inducing accelerated clonal extinction. The overall effect on evolvability would lead to diminished adaptation of pathogenic bacteria in the intestine. Therefore, this newly described non-canonical function of antibodies has very important repercussion for the immune defence. The "enchained growth" process can also explain why, in contrast to serum $\operatorname{IgA}$, the predominant forms of mucosal sIgA are dimers or higher molecular weight polymers (Suzuki et al., 2015). Only such forms of IgA would be proficient in mediating bacterial clumping through "enchained growth". It is tempting to speculate that the same reason explains why, in cases of selective $\operatorname{Ig} \mathrm{A}$ immune deficiencies in human, pentameric $\operatorname{Ig} \mathrm{M}$ and not monomeric IgG compensates for the functions of $\operatorname{IgA}$ in the mucosa.

\section{Non-canonical functions of antibodies important for immune regulation}

\section{Receptor agonistic activity of antibodies}

In a mirror image to antibodies that directly affect gene expression in bacteria and fungi, some antibodies in the human trigger cell signalling upon binding to membrane receptors on endogenous cells, thus assuming the function of receptor ligands (Figure 3). Such antibodies were first identified in patients with different autoimmune diseases and referred to as "functional autoantibodies" (Cabral-Marques and 
Riemekasten, 2017). The main targets of functional autoantibodies are G-coupled protein receptors (GPCRs). This class of cell membrane receptors consists of $>1000$ different members that specifically recognize hormones, chemokines, neuromediators, nucleotides and many other molecules, thus regulating essential physiological processes. Not surprisingly, functional autoantibodies found in patients with autoimmune disorders can have profound physiological impact upon interference with different GPCRs (Cabral-Marques and Riemekasten, 2017).

A recent study demonstrated that the functional antibodies that specifically bind to GPCRs are not anecdotal and merely associated with immune pathologies but are also detected in healthy humans (Cabral-Marques et al., 2018). Thus, antibodies binding to chemokine receptors, acetylcholine receptors, anti-angiotensin II receptor type 1 and $\beta$-adrenergic receptors were detected in sera from healthy individuals. Importantly, the reactivities of antibodies to different types of GPCRs demonstrated strong correlations. In addition, correlations between binding to GPCRs and binding to growth factors or growth factor receptors were also documented. Thus, receptorbinding antibodies establish complex networks of interactions. It was further demonstrated that the particular features of these networks in healthy individuals are influenced by gender and age as well as by different pathological conditions (CabralMarques et al., 2018). In the same study, it was shown that antibodies isolated from healthy individuals that specifically bind to chemokine receptor EDNRA serve as a chemotactic factor for neutrophils. This observation highlights the agonistic activity of GPCRs-specific antibodies, i.e., their ability to induce biological effects similar to those induced by binding of physiological ligands to their cognate receptors. Although the biological repercussion of antibodies that mimic ligands of GPCR has not yet been understood, one can speculate that, at the systemic level, they participate 
in regulation of physiological processes. These antibodies may for instance provide tonic triggers to GPCR receptors and participate in setting physiological thresholds for natural ligands. The nature of the immunological mechanisms at play in the selection and maintenance of B cells producing functional antibodies requires further investigations.

\section{Antibodies counterbalancing congenial immune impairments}

An important non-canonical function of antibodies has recently surfaced upon investigation of patients with genetic impairments of adaptive or innate immunity. Thus, antibodies can compensate for the inherited genetic defects and ameliorate the phenotypic expression in immunodeficiencies (Figure 3).

APS1/APECED is a severe systemic autoimmune syndrome due to functional deficiency of AIRE, a key molecule in central T-cell tolerance. This disorder is usually characterized by autoimmune polyendocrinopathy and candidiasis. Analyses of the reactivity of serum antibodies in patients with APS1/APECED demonstrated the recognition of a large set of human proteins (Fishman et al., 2017, Meyer et al., 2016). On average, each patient showed a strong binding to $>80$ human proteins as compared to ca. 19 in healthy individuals (Meyer et al., 2016). By investigating the immunoreactivity of antibodies of 81 different patients, it was estimated that $>3500$ different human proteins are significantly recognized in patients with APS1/APECED. Interestingly, these analyses also identified a core of overlapping antibody reactivities present in each patient. This core consists of approximately 10 proteins that included important cytokines such as Type I IFNs, IL-22 and IL-17 (Meyer et al., 2016). Further analyses revealed that cytokine-binding autoantibodies harbor high numbers of somatic mutations in their $\mathrm{V}$ genes, recognize conformational epitopes and bind to 
their targets with very high (sub-picomolar) affinities. Importantly, they also show prominent biological activity in cellular assays and animal models. The analysis of clinical manifestations of autoimmunity in APS1/APECED patients uncovered a strong negative correlation between the presence of antibodies against Type I IFNs and development of Type I diabetes. Patients without autoimmune diabetes were able to inhibit the activity of all IFN $\alpha$ variants used in the study. In contrast, APS1/APECED patients who develop Type I diabetes lacked IFN $\alpha$-reactive autoantibodies (Meyer et al., 2016). Together, the results indicate that autoantibodies specific for cytokines that are implicated in autoimmune pathogenesis counterbalance the dramatic lack of self-tolerance consecutive to failure of negative T-cell selection in the thymus. The authors further hypothesized that antibody autoreactivity to Type I IFN and IL-17 explains the relatively low incidence of other autoimmune diseases in APS1/APECED patients such as systemic sclerosis, Sjögren's syndrome, multiple sclerosis and systemic lupus erythematous.

Not unexpectedly and in line with the existence of functionally relevant cytokinebinding autoantibodies, some antibodies may suppress the pathological effects consecutive to congenital impairments of innate immunity (Israel et al., 2017). TIRAP is an adapter molecule associated with TLR receptors. The homozygous mutation R121W results in impaired TLR2 responses, resulting in invasive infections with Staphylococcus aureus. Investigations on the incomplete penetrance of TIRAP deficiency to susceptibility to the infection revealed that most infection-resistant patients produce an antibody against lipoteichoic acid from staphylococcus (Israel et al., 2017). Notably, this antibody restored the deficient lypoteichoic acid-mediated signalling through TLR2/6 and induced IL-6 expression in patients monocytes as well as TIRAP-deficient mouse macrophages. The compensatory mechanism by which 
antibody restores the impaired signalling through TLR2/6 is not understood, but this phenomenon explains the absence of phenotypic expression of the disease in most patients with TIRAP deficiency (Israel et al., 2017).

The existence of antibodies that compensate for inborn immune defects demonstrates the remarkable evolutional plasticity of antibody repertoires. Thus, antibody molecules with appropriate specificities can fill the "gaps" created by impaired immunity. Fundamental questions related to the mechanisms underlying the positive selection and maintenance of B cells producing disease-ameliorating antibodies need to be addressed. A better understanding of these processes shall undoubtedly lead to better therapies for autoimmune diseases and immune deficiencies.

\section{Antibodies as carriers and chaperons}

Antibodies can serve as carriers and chaperons of biologically active molecules

(Figure 3). All healthy humans have antibodies that specifically recognize different peptide hormones, such as ghrelin, $\alpha$-melanocyte stimulating hormone $(\alpha-\mathrm{MSH})$ and adrenocorticotropic hormone (Takagi et al., 2013, Vaeroy et al., 2018). Ghrelin and $\alpha$ MSH are involved in the regulation of eating behaviour, whereas adrenocorticotropic hormone is implicated in stress response. The interaction of antibodies with these hormones are usually characterized by micromolar affinities and thereby cannot compete with the nano or femtomolar affinities that characterize hormone-binding to their cognate receptors (Takagi et al., 2013, Vaeroy et al., 2018). Nevertheless, the formation of complexes with $\mathrm{IgG}$ results in protection of the hormones from proteolytic degradation by plasmatic proteases, thus prolonging their bioavailability (Takagi et al., 2013). Besides, antibody chaperones to $\alpha$-MSH also potentiate its binding to its receptor, the melanocortin 4 receptor. Indeed, in vitro experiments 
revealed that $\alpha-\mathrm{MSH}$ triggers cell signalling considerably more efficiently when complexed with antibodies. Interestingly, the in vivo functional activity of $\alpha$-MSH in reducing appetite (and hence food intake) is abrogated when the hormone is injected in immunodeficient $\left(\mathrm{RAG}^{-/-}\right)$mice that lack endogenous immunoglobulins (Lucas et al., 2019). Whether it is restored in immunoglobulin-reconstituted mice however remains to be investigated. It is noteworthy that the binding kinetics and epitope specificities of ghrelin and $\alpha-\mathrm{MSH}$-specific antibodies are altered in obese patients or patients with eating disorders (Lucas et al., 2019, Takagi et al., 2013). As compared to IgG from healthy individuals, IgG from obese patients provided a better protection of ghrelin from degradation, thus prolonging its biological availability beyond the physiological half-life of the molecule alone (Takagi et al., 2013). In addition, IgG from obese patients and healthy donors recognized different sets of epitopes on $\alpha$ $\mathrm{MSH}$, with the former blocking the interaction of $\alpha-\mathrm{MSH}$ with its receptor (Lucas et al., 2019). Likewise, alterations in binding kinetics and set of targeted epitopes of hormone carrier antibodies specific for the adrenocorticotropic hormone were also associated with changes in the behaviour of humans (Vaeroy et al., 2018).

Taken together these finding indicate that antibodies can play auxiliary role in the activity of physiologically active peptides. It is possible that this non-canonical function of immunoglobulins concerns much larger set of molecules that presently identified.

\section{Antibodies as modulators of cell signalling}

A recent investigation highlighted a novel mechanism of hormone regulation by antibodies that exclusively relies on immunoglobulin constant regions (Figure 3). Tanigaki et al. (Tanigaki et al., 2018) observed that systemic or endothelium-specific 
deficiency in FcyRIIb protects mice from obesity-induced insulin resistance and decrease in skeletal muscle glucose uptake. The selective deficiency in IgG also protected the animals from the development of insulin resistance, indicating that the interaction of endothelial FcyRIIb with IgG contributes to the pathological process. Importantly, glucose intolerance and insulin resistance was transferred in IgGdeficient mice fed with high fat diet following reconstitution with pooled $\operatorname{IgG}$ from Type II diabetes mellitus patients, but not with IgG obtained from healthy individuals. Further mechanistic studies demonstrated that interactions of IgG with FcyRIIb expressed on endothelial cells inhibits insulin induced-cell signalling that depends on eNOS and ultimately reduces transcytosis of insulin (Tanigaki et al., 2018). The modulation of insulin signalling was induced by a specific glycoform of $\operatorname{IgG}$ that was characterized with low levels of sialic acids on the Asn297 glycan structure (Box 2). Hypo-sialyalated $\operatorname{IgG}$ appeared in obese mice and was detected also in diabetes patients. Importantly, hypo-sialylated IgG was found to bind to Fc $\gamma$ RIIb with 10-fold higher affinity as compared to other IgG glycoforms (Tanigaki et al., 2018). The high affinity may allow binding to Fc $\gamma$ RIIb expressed on endothelial cells in absence of crosslinking by antigen, thus resulting in specific signalling that interferes with insulin physiological activities.

\section{Concluding remarks}

In the present review, we provided examples of different activities of antibodies that breach the paradigm about biological functions of antibodies. The examples of noncanonical functions of antibodies enrich our understanding about of the adaptive immune response by revealing different ingenious strategies developed by the immune system to ultimate diversify its operational capability. With these multitude of activities (Figure 2 and 3 ), antibodies emerge as one of the most functionally 
versatile class of glycoproteins. Importantly, most of the described non-conventional functions of antibodies are constructively present in healthy individuals whereas other appears only in cases of disturbed homeostasis (i.e., compensatory function for immunodeficiency).

The non-canonical functions of antibodies contribute both to immune defence and immune regulation. While for the sake of clarity, these two categories were discussed separately in the present review, many non-canonical functions may contribute to both. For example, catalytic antibodies can neutralize pathogens but also activate some coagulation molecules, thus compensating for certain coagulopathies. Similarly, the control of the penetration of metabolites by intestinal bacteria contributes to the regulation of immune defence function, but also plays a key role in regulating host homeostasis.

Antibodies are generated as a result of microevolution processes of mutation and selection, and hence their repertoires are very adaptable and dynamic. Thus, the vast diversity and evolutionary selection forces may sustain the emergence of most of the non-canonical functions of antibodies. Indeed, most of these functions are executed via the variable region of antibodies. Since most of the non-canonical functions of antibodies arise as results of stochastic processes of diversification and evolutionary selection, we are tempted to speculate that future studies will unfold many other stratagems that adaptive immune response utilizes to confer antibodies with functional activities that extend well beyond the classical triad antigen-antibodyinnate receptor. 


\section{Acknowledgments}

This work was supported by Institut National de la Santé et de la Recherche Médicale (INSERM, France) and by the European Research Council (Project CoBABATI ERCStG-678905 to J.D.D.).

\section{References}

1. Abboud, N., Chow, S.K., Saylor, C., Janda, A., Ravetch, J.V., Scharff, M.D., and Casadevall, A. (2010) A requirement for FcgammaR in antibody-mediated bacterial toxin neutralization. The Journal of experimental medicine 207, 2395-2405.

2. Benckert, J., Schmolka, N., Kreschel, C., Zoller, M.J., Sturm, A., Wiedenmann, B., and Wardemann, H. (2011) The majority of intestinal IgA+ and $\mathrm{IgG}+$ plasmablasts in the human gut are antigen-specific. The Journal of clinical investigation 121, 1946-1955.

3. Berkowska, M.A., Schickel, J.N., Grosserichter-Wagener, C., de Ridder, D., Ng, Y.S., van Dongen, J.J., . . . van Zelm, M.C. (2015) Circulating Human CD27-IgA+ Memory B Cells Recognize Bacteria with Polyreactive Igs. Journal of immunology 195, 1417-1426.

4. Bournazos, S., Chow, S.K., Abboud, N., Casadevall, A., and Ravetch, J.V. (2014a) Human IgG Fc domain engineering enhances antitoxin neutralizing antibody activity. The Journal of clinical investigation 124, 725-729.

5. Bournazos, S., Klein, F., Pietzsch, J., Seaman, M.S., Nussenzweig, M.C., and Ravetch, J.V. (2014b) Broadly neutralizing anti-HIV-1 antibodies require Fc effector functions for in vivo activity. Cell 158, 1243-1253.

6. Bournazos, S., Wang, T.T., Dahan, R., Maamary, J., and Ravetch, J.V. (2017) Signaling by Antibodies: Recent Progress. Annual review of immunology 35, 285-311.

7. Bowen, A., Wear, M., and Casadevall, A. (2017a) Antibody-Mediated Catalysis in Infection and Immunity. Infection and immunity 85. 
8. Bowen, A., Wear, M.P., Cordero, R.J., Oscarson, S., and Casadevall, A. (2017b) A Monoclonal Antibody to Cryptococcus neoformans Glucuronoxylomannan Manifests Hydrolytic Activity for Both Peptides and Polysaccharides. The Journal of biological chemistry 292, 417-434.

9. Bunker, J.J., Erickson, S.A., Flynn, T.M., Henry, C., Koval, J.C., Meisel, M., . . . Bendelac, A. (2017) Natural polyreactive IgA antibodies coat the intestinal microbiota. Science 358.

10. Bunker, J.J., Flynn, T.M., Koval, J.C., Shaw, D.G., Meisel, M., McDonald, B.D., . . . Bendelac, A. (2015) Innate and Adaptive Humoral Responses Coat Distinct Commensal Bacteria with Immunoglobulin A. Immunity 43, 541-553.

11. Cabral-Marques, O., Marques, A., Giil, L.M., De Vito, R., Rademacher, J., Gunther, J., . . . Riemekasten, G. (2018) GPCR-specific autoantibody signatures are associated with physiological and pathological immune homeostasis. Nature communications 9, 5224.

12. Cabral-Marques, O. and Riemekasten, G. (2017) Functional autoantibodies targeting $\mathrm{G}$ protein-coupled receptors in rheumatic diseases. Nature reviews. Rheumatology 13, 648-656.

13. Casadevall, A. and Pirofski, L.A. (2011) A new synthesis for antibodymediated immunity. Nature immunology 13, 21-28.

14. Coleman, J.L., Rogers, R.C., and Benach, J.L. (1992) Selection of an escape variant of Borrelia burgdorferi by use of bactericidal monoclonal antibodies to OspB. Infection and immunity 60, 3098-3104.

15. Connolly, S.E., Thanassi, D.G., and Benach, J.L. (2004) Generation of a complement-independent bactericidal IgM against a relapsing fever Borrelia. Journal of immunology 172, 1191-1197.

16. Diebolder, C.A., Beurskens, F.J., de Jong, R.N., Koning, R.I., Strumane, K., Lindorfer, M.A., . . . Parren, P.W. (2014) Complement is activated by IgG hexamers assembled at the cell surface. Science 343, 1260-1263.

17. Dimitrov, J.D., Planchais, C., Roumenina, L.T., Vassilev, T.L., Kaveri, S.V., and Lacroix-Desmazes, S. (2013) Antibody polyreactivity in health and disease: statu variabilis. Journal of immunology 191, 993-999.

18. Donaldson, G.P., Ladinsky, M.S., Yu, K.B., Sanders, J.G., Yoo, B.B., Chou, W.C., . . Mazmanian, S.K. (2018) Gut microbiota utilize immunoglobulin A for mucosal colonization. Science 360, 795-800. 
19. Fishman, D., Kisand, K., Hertel, C., Rothe, M., Remm, A., Pihlap, M., . . . Peterson, P. (2017) Autoantibody Repertoire in APECED Patients Targets Two Distinct Subgroups of Proteins. Frontiers in immunology 8, 976.

20. Fransen, F., Zagato, E., Mazzini, E., Fosso, B., Manzari, C., El Aidy, S., . . Rescigno, M. (2015) BALB/c and C57BL/6 Mice Differ in Polyreactive IgA Abundance, which Impacts the Generation of Antigen-Specific IgA and Microbiota Diversity. Immunity 43, 527-540.

21. Hendrickx, A.P., Top, J., Bayjanov, J.R., Kemperman, H., Rogers, M.R., Paganelli, F.L., . . . Willems, R.J. (2015) Antibiotic-Driven Dysbiosis Mediates Intraluminal Agglutination and Alternative Segregation of Enterococcus faecium from the Intestinal Epithelium. mBio 6, e01346-01315.

22. Hifumi, E., Morihara, F., Hatiuchi, K., Okuda, T., Nishizono, A., and Uda, T. (2008) Catalytic features and eradication ability of antibody light-chain UA15L against Helicobacter pylori. The Journal of biological chemistry 283, 899907.

23. Israel, L., Wang, Y., Bulek, K., Della Mina, E., Zhang, Z., Pedergnana, V., . . Puel, A. (2017) Human Adaptive Immunity Rescues an Inborn Error of Innate Immunity. Cell 168, 789-800 e710.

24. Kanyavuz, A., Marey-Jarossay, A., Lacroix-Desmazes, S., and Dimitrov, J.D. (2019) Breaking the law: unconventional strategies for antibody diversification. Nature reviews. Immunology 19, 355-368.

25. Lacroix-Desmazes, S., Bayry, J., Kaveri, S.V., Hayon-Sonsino, D., Thorenoor, N., Charpentier, J., . . Mallet, V.O. (2005) High levels of catalytic antibodies correlate with favorable outcome in sepsis. Proceedings of the National Academy of Sciences of the United States of America 102, 4109-4113.

26. Lacroix-Desmazes, S., Bayry, J., Misra, N., Horn, M.P., Villard, S., Pashov, A., . . Kaveri, S.V. (2002) The prevalence of proteolytic antibodies against factor VIII in hemophilia A. The New England journal of medicine 346, 662667.

27. LaRocca, T.J., Crowley, J.T., Cusack, B.J., Pathak, P., Benach, J., London, E., . . . Benach, J.L. (2010) Cholesterol lipids of Borrelia burgdorferi form lipid rafts and are required for the bactericidal activity of a complementindependent antibody. Cell host \& microbe 8, 331-342. 
28. LaRocca, T.J., Holthausen, D.J., Hsieh, C., Renken, C., Mannella, C.A., and Benach, J.L. (2009) The bactericidal effect of a complement-independent antibody is osmolytic and specific to Borrelia. Proceedings of the National Academy of Sciences of the United States of America 106, 10752-10757.

29. LaRocca, T.J., Katona, L.I., Thanassi, D.G., and Benach, J.L. (2008) Bactericidal action of a complement-independent antibody against relapsing fever Borrelia resides in its variable region. Journal of immunology 180, 62226228.

30. Li, L., Paul, S., Tyutyulkova, S., Kazatchkine, M.D., and Kaveri, S. (1995) Catalytic activity of anti-thyroglobulin antibodies. Journal of immunology 154, 3328-3332.

31. Lu, L.L., Suscovich, T.J., Fortune, S.M., and Alter, G. (2018) Beyond binding: antibody effector functions in infectious diseases. Nature reviews. Immunology 18, 46-61.

32. Lucas, N., Legrand, R., Bole-Feysot, C., Breton, J., Coeffier, M., Akkermann, K., . . Fetissov, S.O. (2019) Immunoglobulin G modulation of the melanocortin 4 receptor signaling in obesity and eating disorders. Translational psychiatry $9,87$.

33. Macpherson, A.J., Yilmaz, B., Limenitakis, J.P., and Ganal-Vonarburg, S.C. (2018) IgA Function in Relation to the Intestinal Microbiota. Annual review of immunology 36, 359-381.

34. McClelland, E.E., Nicola, A.M., Prados-Rosales, R., and Casadevall, A. (2010) Ab binding alters gene expression in Cryptococcus neoformans and directly modulates fungal metabolism. The Journal of clinical investigation 120, 1355-1361.

35. McEwan, W.A., Tam, J.C., Watkinson, R.E., Bidgood, S.R., Mallery, D.L., and James, L.C. (2013) Intracellular antibody-bound pathogens stimulate immune signaling via the Fc receptor TRIM21. Nature immunology 14, 327336.

36. Meyer, S., Woodward, M., Hertel, C., Vlaicu, P., Haque, Y., Karner, J., . . . Hayday, A. (2016) AIRE-Deficient Patients Harbor Unique High-Affinity Disease-Ameliorating Autoantibodies. Cell 166, 582-595. 
37. Mkaddem, S.B., Christou, I., Rossato, E., Berthelot, L., Lehuen, A., and Monteiro, R.C. (2014) IgA, IgA receptors, and their anti-inflammatory properties. Current topics in microbiology and immunology 382, 221-235.

38. Moor, K., Diard, M., Sellin, M.E., Felmy, B., Wotzka, S.Y., Toska, A., . . . Slack, E. (2017) High-avidity IgA protects the intestine by enchaining growing bacteria. Nature 544, 498-502.

39. Mukherjee, J., Nussbaum, G., Scharff, M.D., and Casadevall, A. (1995) Protective and nonprotective monoclonal antibodies to Cryptococcus neoformans originating from one B cell. The Journal of experimental medicine 181, 405-409.

40. Paul, S., Karle, S., Planque, S., Taguchi, H., Salas, M., Nishiyama, Y., . . . Hanson, C. (2004) Naturally occurring proteolytic antibodies: selective immunoglobulin M-catalyzed hydrolysis of HIV gp120. The Journal of biological chemistry 279, 39611-39619.

41. Paul, S., Volle, D.J., Beach, C.M., Johnson, D.R., Powell, M.J., and Massey, R.J. (1989) Catalytic hydrolysis of vasoactive intestinal peptide by human autoantibody. Science 244, 1158-1162.

42. Planque, S., Mitsuda, Y., Taguchi, H., Salas, M., Morris, M.K., Nishiyama, Y., . . Paul, S. (2007) Characterization of gp120 hydrolysis by IgA antibodies from humans without HIV infection. AIDS research and human retroviruses $23,1541-1554$.

43. Planque, S.A., Nishiyama, Y., Sonoda, S., Lin, Y., Taguchi, H., Hara, M., . . Paul, S. (2015) Specific amyloid beta clearance by a catalytic antibody construct. The Journal of biological chemistry 290, 10229-10241.

44. Ponomarenko, N.A., Durova, O.M., Vorobiev, II, Belogurov, A.A., Jr., Kurkova, I.N., Petrenko, A.G., . . Gabibov, A.G. (2006) Autoantibodies to myelin basic protein catalyze site-specific degradation of their antigen. Proceedings of the National Academy of Sciences of the United States of America 103, 281-286.

45. Pradhan, V., Pandit, P., Surve, P., Lecerf, M., Rajadhyaksha, A., Nadkar, M., . . Kaveri, S.V. (2018) Catalytic antibodies in patients with systemic lupus erythematosus. European journal of rheumatology 5, 173-178.

46. Rochereau, N., Drocourt, D., Perouzel, E., Pavot, V., Redelinghuys, P., Brown, G.D., . . . Paul, S. (2013) Dectin-1 is essential for reverse transcytosis of 
glycosylated SIgA-antigen complexes by intestinal M cells. PLoS biology 11, e1001658.

47. Sadziene, A., Jonsson, M., Bergstrom, S., Bright, R.K., Kennedy, R.C., and Barbour, A.G. (1994) A bactericidal antibody to Borrelia burgdorferi is directed against a variable region of the OspB protein. Infection and immunity 62, 2037-2045.

48. Saphire, E.O., Parren, P.W., Pantophlet, R., Zwick, M.B., Morris, G.M., Rudd, P.M., . . . Wilson, I.A. (2001) Crystal structure of a neutralizing human IGG against HIV-1: a template for vaccine design. Science 293, 1155-1159.

49. Schroeder, H.W., Jr. and Cavacini, L. (2010) Structure and function of immunoglobulins. The Journal of allergy and clinical immunology 125, S4152.

50. Sharp, T.H., Boyle, A.L., Diebolder, C.A., Kros, A., Koster, A.J., and Gros, P. (2019) Insights into IgM-mediated complement activation based on in situ structures of IgM-C1-C4b. Proceedings of the National Academy of Sciences of the United States of America 116, 11900-11905.

51. Storek, K.M., Auerbach, M.R., Shi, H., Garcia, N.K., Sun, D., Nickerson, N.N., . . . Rutherford, S.T. (2018) Monoclonal antibody targeting the betabarrel assembly machine of Escherichia coli is bactericidal. Proceedings of the National Academy of Sciences of the United States of America 115, 3692-3697.

52. Suzuki, T., Kawaguchi, A., Ainai, A., Tamura, S., Ito, R., Multihartina, P., . . Hasegawa, H. (2015) Relationship of the quaternary structure of human secretory IgA to neutralization of influenza virus. Proceedings of the National Academy of Sciences of the United States of America 112, 7809-7814.

53. Takagi, K., Legrand, R., Asakawa, A., Amitani, H., Francois, M., Tennoune, N., . . Fetissov, S.O. (2013) Anti-ghrelin immunoglobulins modulate ghrelin stability and its orexigenic effect in obese mice and humans. Nature communications 4, 2685.

54. Tanigaki, K., Sacharidou, A., Peng, J., Chambliss, K.L., Yuhanna, I.S., Ghosh, D., . . . Mineo, C. (2018) Hyposialylated IgG activates endothelial IgG receptor FcgammaRIIB to promote obesity-induced insulin resistance. The Journal of clinical investigation 128, 309-322.

55. Tian, H., Weber, S., Thorkildson, P., Kozel, T.R., and Pirofski, L.A. (2009) Efficacy of opsonic and nonopsonic serotype 3 pneumococcal capsular 
polysaccharide-specific monoclonal antibodies against intranasal challenge with Streptococcus pneumoniae in mice. Infection and immunity 77, 15021513.

56. Tiller, T., Tsuiji, M., Yurasov, S., Velinzon, K., Nussenzweig, M.C., and Wardemann, H. (2007) Autoreactivity in human IgG+ memory B cells. Immunity 26, 205-213.

57. Uchimura, Y., Fuhrer, T., Li, H., Lawson, M.A., Zimmermann, M., Yilmaz, B., . . . Macpherson, A.J. (2018) Antibodies Set Boundaries Limiting Microbial Metabolite Penetration and the Resultant Mammalian Host Response. Immunity 49, 545-559 e545.

58. Vaeroy, H., Adori, C., Legrand, R., Lucas, N., Breton, J., Cottard, C., . . . Fetissov, S.O. (2018) Autoantibodies reactive to adrenocorticotropic hormone can alter cortisol secretion in both aggressive and nonaggressive humans. Proceedings of the National Academy of Sciences of the United States of America 115, E6576-E6584.

59. van Egmond, M., van Garderen, E., van Spriel, A.B., Damen, C.A., van Amersfoort, E.S., van Zandbergen, G., . . . van de Winkel, J.G. (2000) FcalphaRI-positive liver Kupffer cells: reappraisal of the function of immunoglobulin A in immunity. Nature medicine 6, 680-685.

60. Vidarsson, G., Dekkers, G., and Rispens, T. (2014) IgG subclasses and allotypes: from structure to effector functions. Frontiers in immunology 5, 520.

61. Wootla, B., Christophe, O.D., Mahendra, A., Dimitrov, J.D., Repesse, Y., Ollivier, V., . . . Lacroix-Desmazes, S. (2011) Proteolytic antibodies activate factor IX in patients with acquired hemophilia. Blood 117, 2257-2264.

62. Wootla, B., Dasgupta, S., Dimitrov, J.D., Bayry, J., Levesque, H., Borg, J.Y., . . . Lacroix-Desmazes, S. (2008) Factor VIII hydrolysis mediated by anti-factor VIII autoantibodies in acquired hemophilia. Journal of immunology $180,7714-7720$.

63. Xu, Y., Yamamoto, N., and Janda, K.D. (2004) Catalytic antibodies: hapten design strategies and screening methods. Bioorganic \& medicinal chemistry $12,5247-5268$.

64. Yang, Y.H., Chang, C.J., Chuang, Y.H., Hsu, H.Y., Chen, P.P., and Chiang, B.L. (2010) Identification of anti-prothrombin antibodies in the anti- 
phospholipid syndrome that display the prothrombinase activity. Rheumatology 49, 34-42.

65. Yano, M., Gohil, S., Coleman, J.R., Manix, C., and Pirofski, L.A. (2011) Antibodies to Streptococcus pneumoniae capsular polysaccharide enhance pneumococcal quorum sensing. mBio 2.

\section{BOX 1. Structural organization of antibodies}

Antibodies are glycoproteins (immunoglobulins) that exist both in soluble and in membrane-bound form. Immunoglobulins adopt a quaternary structure that consists of polypeptide chains with distinct molecular weights (referred to as light and heavy chains). A number of bisulfide bonds and non-covalent interactions hold together two identical heavy and two identical light chains, resulting in the archetypical Y-shaped immunoglobulin unit (Figure). The light chains fold in two whereas the heavy chains in four (or five) immunoglobulin domains; each domain consists of 110-130 amino acid residues and assumes a characteristic $\beta$-sheet structure.

This diversity of antibodies arises from sequence variations in the $\mathrm{N}$-terminal portion of heavy and light immunoglobulin chains. Owing to the substantial sequence differences between different antibody molecules, the immunoglobulin domains located at the N-terminal ends of heavy and light chains are termed variable (V) domains. The remaining immunoglobulin domains are characterized with low degree of sequence variability and hence are dubbed - constant $(\mathrm{C})$. Within $\mathrm{V}$ domains the sequence diversity is not evenly distributed. Thus, there are regions characterized by particularly high levels of variability i.e. complementarity determining regions, (CDRs). On the other hand there are parts of the $\mathrm{V}$ domain characterized by a lower 
degree of sequence variability that are referred to as framework regions. CDRs of heavy and light chains are brought in a close proximity in the intact antibody structure, thus forming the antigen-binding site.

\section{BOX 2. Heterogeneity of Fc portion of IgG}

Antibodies belonging to $\mathrm{IgG}$ class have a conservative bi-antennary $\mathrm{N}$-glycan structure attached to Asn297 on the $\mathrm{CH} 2$ domain. It was demonstrated that more than 30 alternative glycan variants are displayed on this site. In addition, IgG1 and IgG3 have 4 and 13 polymorphic forms (allotypes), respectively, which are expressed differently in different human populations. These variants in glycan structure or amino acid sequence within a single immunoglobulin subclass strongly impact the antibody affinity for Fc $\gamma$ receptors and C1q. Moreover, some glycoforms permit binding to glycan-binding receptors (or Type II Fc receptors) such as CD22, and DCSIGN, resulting in induction of distinct biological reactions. Since the expression of different classes, subclasses and glycoforms of Fc is a very dynamic process, regulated by the tissue microenvironment, the heterogeneity of the constant regions provides a high level of plasticity of effector functions for antibodies.

\section{Figure legends}

Figure 1. Conventional functions of antibodies. Most of the classical functions of antibodies depend on formation of the tertiary complex: antigen - antibody - innate 
receptor. The innate receptors are diverse and are expressed at the surface of different immune and non-immune cells or are soluble $(\mathrm{C} 1 \mathrm{q})$. The engagement of the innate receptors by the complex antigen-antibody can trigger different processes, referred to as effector functions. The concrete effector function depends on the particular type of innate receptor, cell type and properties of antibodies.

Figure 2. Non-conventional functions of antibodies that contribute to immune defence. These functions of antibodies depend exclusively on the sequence characteristics of variable region. Antibodies can bind to surface of microbes and induce direct killing through different molecular mechanisms. Antibodies with catalytic activity can contribute to immune defence by hydrolysing macromolecules important for pathogens. High-avidity IgA antibodies specific to intestinal microbes can trigger formation of bacterial clusters by a process defined as "enchained bacterial growth". This result in a faster intestinal transfer of bacteria, reduction of clonal diversity and inhibition of gene transfer between bacteria. IgA antibodies can also control the penetration of bacterial metabolites in the host. This function might contribute both to immune defence and immune regulation. Finally, non-specific (polyreactive) IgA are able to facilitate the trans-epithelial transfer of bacteria. This process results in induction of specific mucosal immune response and production of high-affinity specific antibodies that may contribute to regulation of bacterial colonization and maintenance of the intestinal bacterial diversity.

Figure 3. Non-conventional functions of antibodies that contribute to immune regulation. Antibodies can bind via their antigen-binding sites to cellular receptors (as 
for example G-protein coupled receptors) and act as agonistic molecules. Antibodies can also recognize peptide hormones and protect them from degradation or fast clearance. These antibodies can also facilitate the signalling induced by some hormones. In case of severe impairments of innate or adaptive immunity in humans certain antibodies directed against cytokines or microbial products can compensate (buffer) the defects in the immune systems. All these functions of antibodies depend on the unique sequence characteristics of variable domain of antibodies. Beside noncanonical functions that depend exclusively on $\mathrm{V}$ region, particular changes in glycan composition at the conservative glycosylation site (Asn297) on Fc can alter interaction of IgG antibodies with FcyRIIB, expressed on endothelial cells. This interaction interferes with transcytosis of insulin and access of the latter to muscle cells.

Figure BOX1. Structural organization of human IgG1 molecule. The fragment of an antibody molecule consisting of $\mathrm{V}$ domains (coloured in pink) and the $\mathrm{C}_{\mathrm{H} 1}$ and the constant domain $(\mathrm{C} \kappa$ or $\mathrm{C} \lambda$ ) of light chain is referred to as Fab (fragment antigen binding). The C-terminal parts of immunoglobulin heavy and light chains have considerably lower sequence diversity as compared to $\mathrm{V}$ region. The portion of the antibody molecule composed by C-terminal segments of heavy chain is defined as constant fragment (Fc, depicted in blue). The structural model of IgG1 was build with UCSF Chimera software, using PDB file 1HZH from (Saphire et al., 2001). 
Figure 1

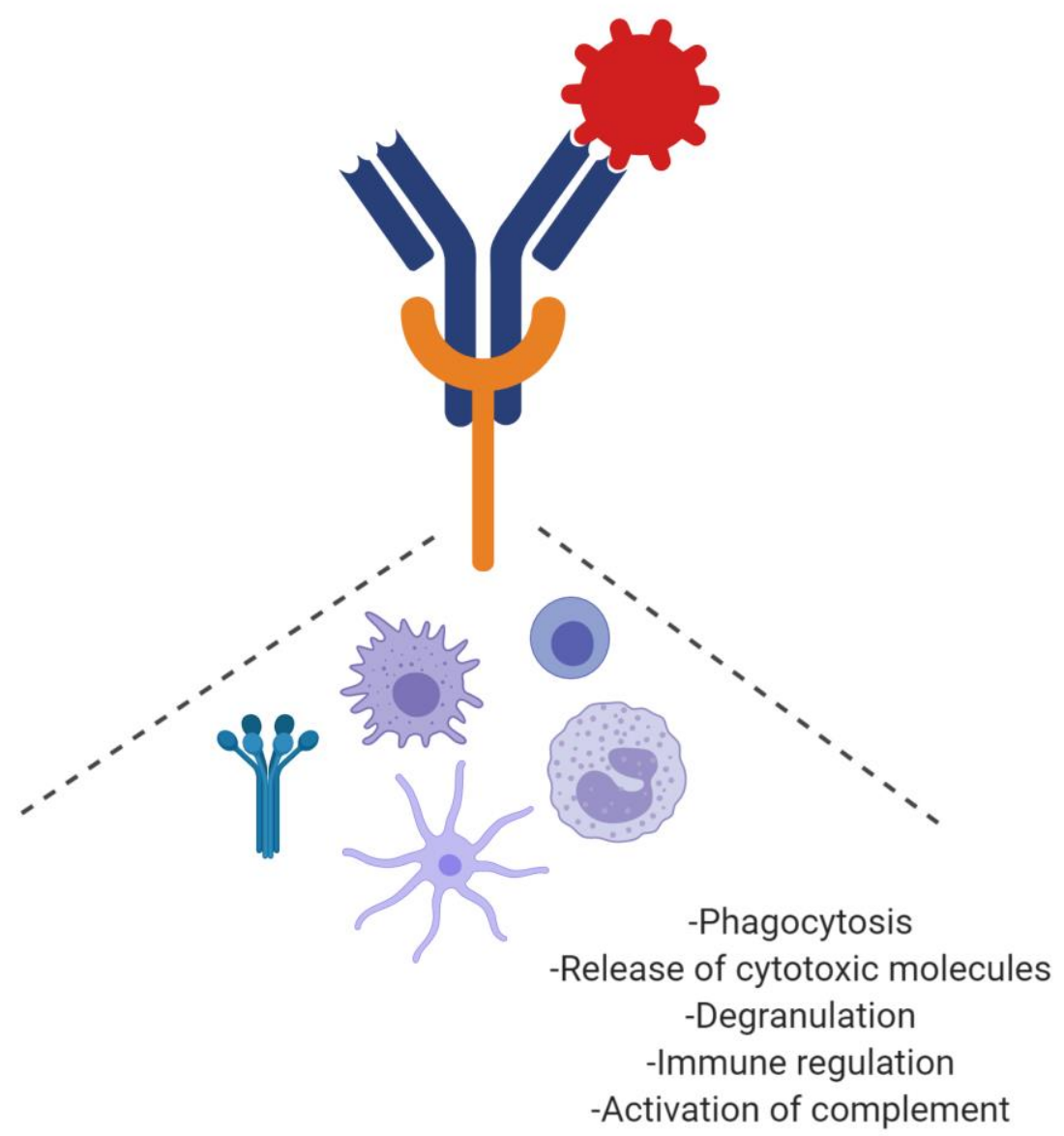


Figure 2

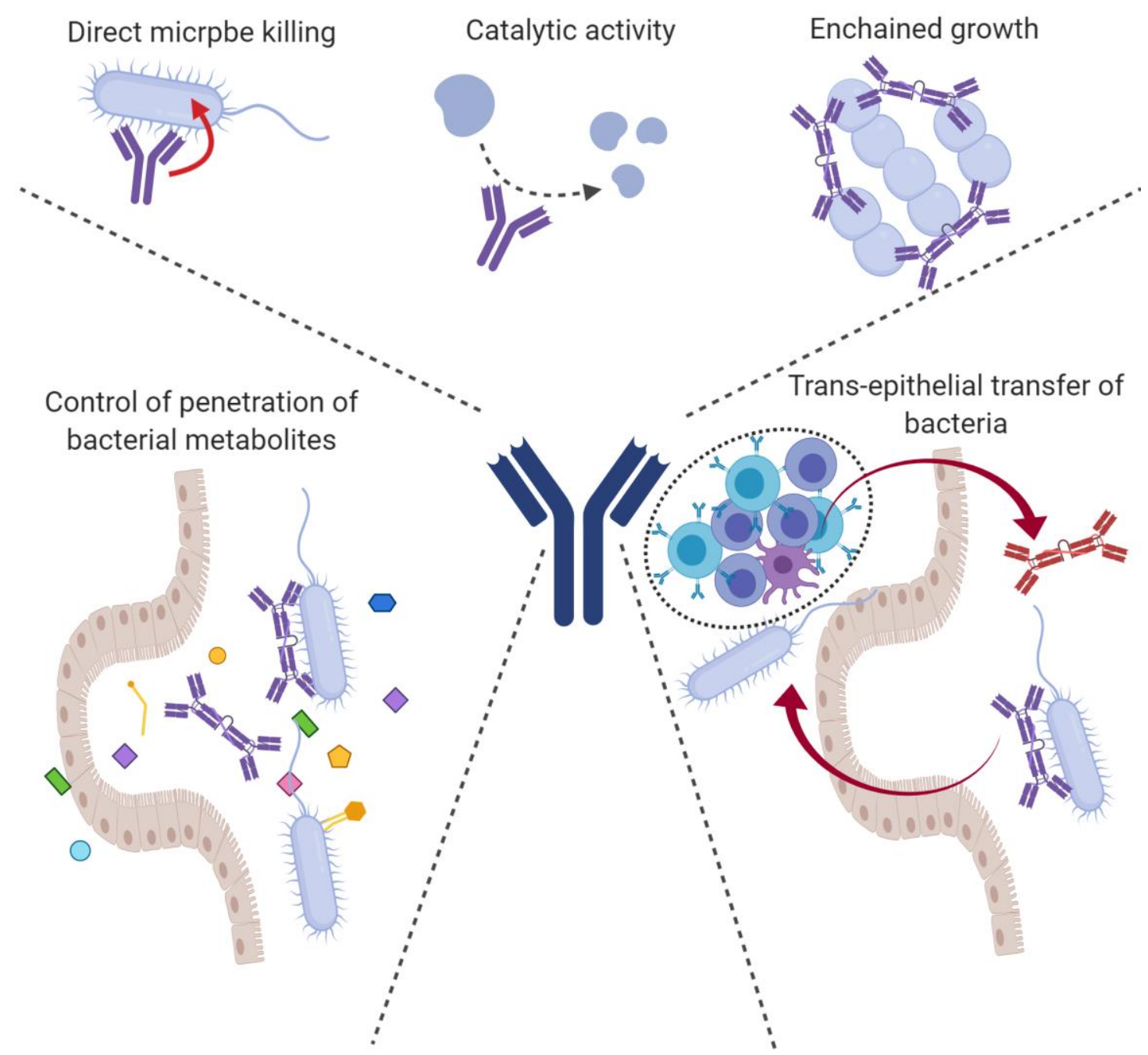


Figure 3

Transporter / chaperone activity
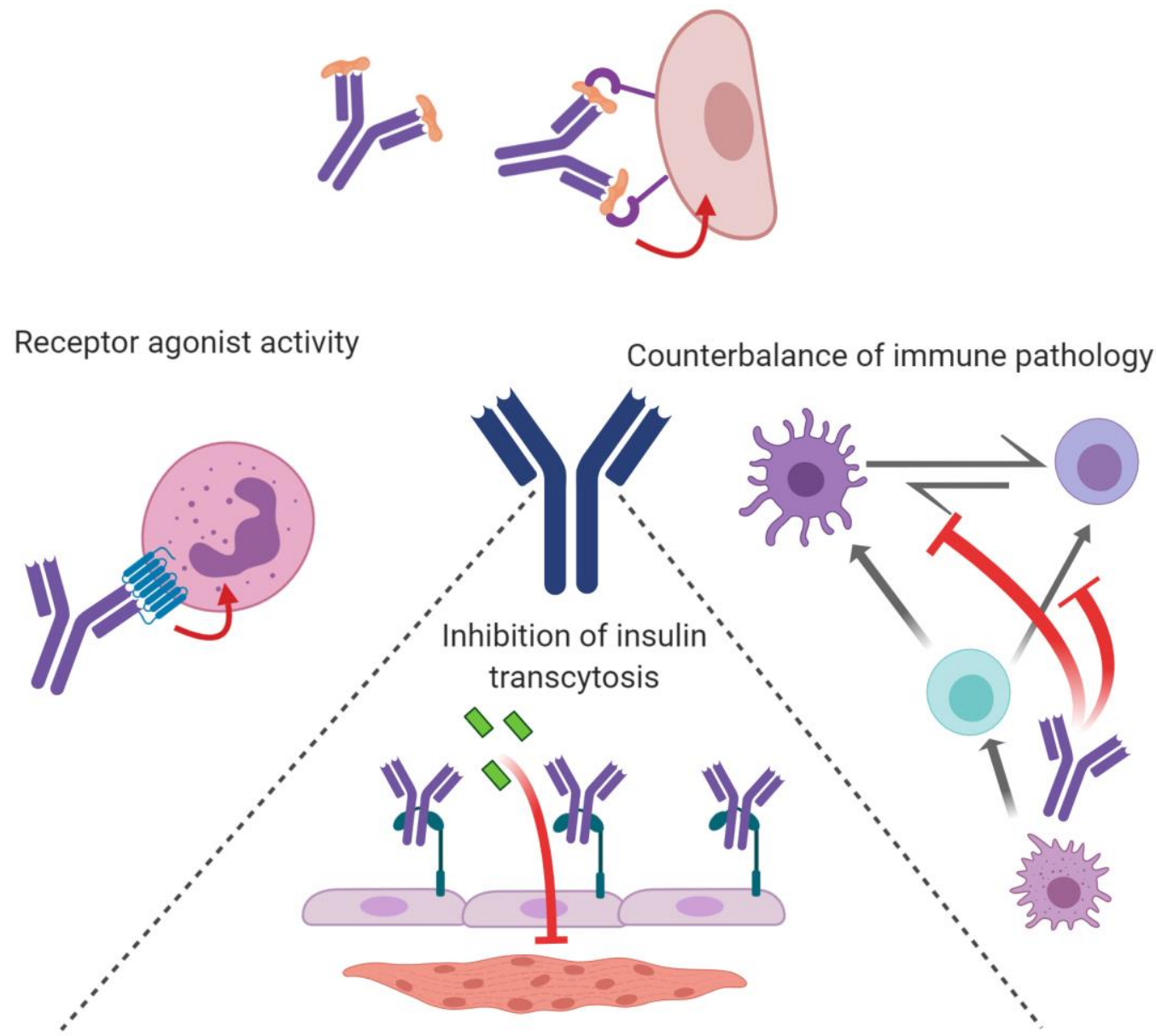
Figure BOX

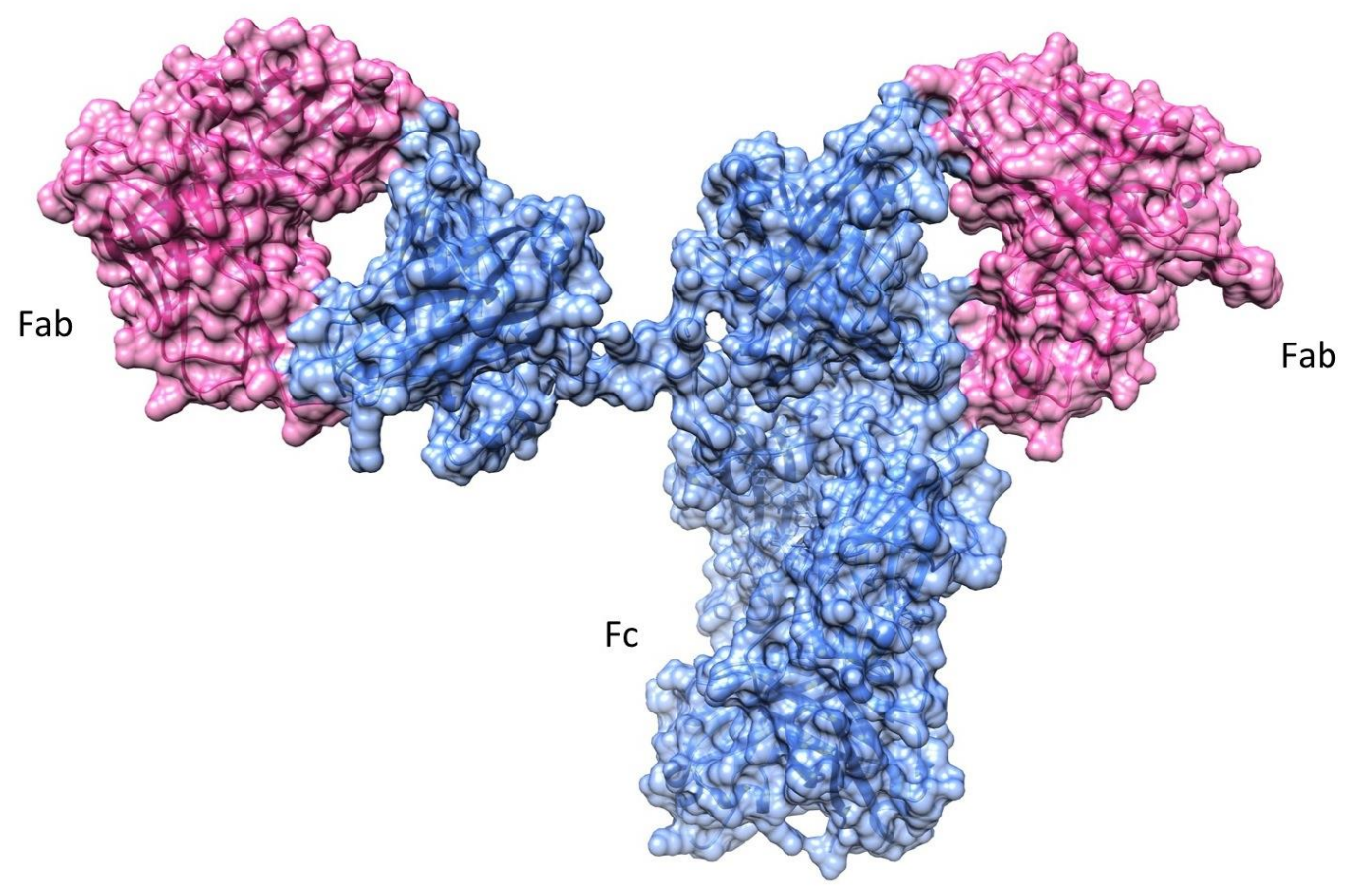




\section{Outstanding questions}

As some of the non-conventional functions of antibodies frequently depend on recognition of endogenous molecules, it remains not clear what are the mechanisms of selection of and maintenance of clones of antibodies with untypical properties.

There are some clues that certain non-conventional functions of antibodies arise as consequence of prominent antigen-binding polyreactivity. The role of polyreactivity in determining untypical functional activity of antibodies remains to be evaluated.

The fact that some antibodies present with functions that are typical for other biologically important molecules, results that some of non-conventional functions can have implications beyond immune system. The role of antibodies as messengers in the cross talk between immune, nervous or endocrine system needs to be deeply investigated.

What is the prevalence in health and disease of antibodies that utilize non-canonical functions in humans? Are these functions originate from specific B cell subpopulation?

How can we exploit the non-canonical functions of antibodies as therapeutic agents, diagnostic markers and laboratory tools? 